\title{
Il buttonhole: come trasformare una fistola in un catetere
}

\author{
Marcello Napoli
}

\author{
U.O.C. Nefrologia e Dialisi, P.O. S. Caterina Novella, Galatina (LE)
}

\begin{abstract}
The BUTTONHOLE TEChNiQUe: HOW TO CONVERT A FISTUla INTO A CATHETER
Abstract. The buttonhole technique (BT) is an alternative to the standard needling for the cannulation of arterio-venous fistula (AVF). The balance between benefits and harms associated with the BT is unclear.

In order to analyze the recent literature we searched PubMed for "randomized controlled study on buttonhole" and "review of buttonhole cannulation", published between January 2011 and November 2013.

We identified 3 randomized trials and 3 reviews. One randomized trial showed a better survival of the AVFs (100\% vs $82 \%)$, fewer interventions (19\% vs 39\%), and lower growth of pre-existing aneurysms $(23 \%$ vs $67 \%)$ in the BT group. A second study showed a greater number of infections and hematomas, and a greater perception of pain in the BT group. A third study showed no differences in perception of pain and bleeding time between the BT group and the control group; additionally, it reported a greater number of hematomas in the control group and a greater number of local infections and sepsis in the BT group. The three reviews showed no definite results regarding the various endpoints of $\mathrm{BT}$ (perception of pain, aneurysm formation, bleeding time), and confirmed the increased risk of local and systemic infections.

Based on this analysis, we can conclude that the BT produces an increased risk of local and systemic infections without definite benefits. Recent studies suggest reserving the BT in selected cases (vessels particularly deep or too short stretches). However, we cannot exclude that further randomized controlled trials may lead to different conclusions on the BT.
\end{abstract}

Key words: Vascular Access, Bottonhole, Cannulation of arterio-venous fistula

Conflict of interest: None.

Financial support: None.

Ricevuto: 9 Novembre 2013; Accettato: 2 Dicembre 2013

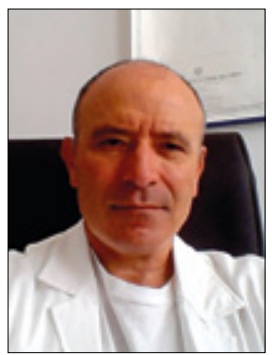

Marcello Napoli

\section{Introduzione}

La BT (puntura ad asola) è una modalità di venipuntura delle fistole artero-venose (FAV) per emodialisi che, messa a punto casualmente più di 30 anni fa (1), recentemente è stata rispolverata tra l'entusiasmo dei suoi sostenitori, con la comparsa di diverse pubblicazioni che ne descrivono l'efficacia e l'efficienza (2-4). La BT è alternativa sia alla tecnica a scala di corda (venipuntura in punti diversi a rotazione) (5) sia alla tecnica ad area, che prevede la venipuntura sempre nella stessa sede (6). La BT consiste nel pungere sempre nello stesso punto i vasi efferenti di una FAV, inizialmente con un ago tagliente, creando, nel volgere di alcune settimane, un vero e proprio tunnel sottocutaneo che, poi, funge da guida per l'incannulamento del vaso. Alcuni Autori si sono avvalsi di un tunnellizzatore che veniva tenuto in sede tra una puntura e l'altra proprio al fine di facilitare la formazione del tunnel (7). Altra prerogativa della BT è rappresentata dal fatto che, una volta creato il tunnel, la venipuntura viene attuata con un ago smusso, con la conseguente potenziale riduzione della percezione del dolore. I primi report sono stati particolarmente positivi, dimostrando la superiorità della BT rispetto alla tecnica a scala di corda, riguardo alla formazione di stenosi, aneurismi e trombosi, oltre alla presenza di una minore percezione del dolore (2-4). Un aspetto negativo era rappresentato da un aumento delle infezioni prodotte verosimilmente dall'inoculazione di germi presenti nel tunnel durante il passaggio dell'ago. Contemporaneamente, si è concretizzata una letteratura in cui i vantaggi della BT nei confronti della tecnica a scala di corda non erano così evidenti $(8,9)$, generando un po' di confusione sia tra gli utilizzatori che tra quelli che si accingevano a sviluppare un'esperienza con la BT. Allo scopo di analizzare la letteratura recente, abbiamo ricercato su PubMed la voce "randomized controlled study on buttonhole" e la voce "review of buttonhole cannulation" negli anni tra il Gennaio 2011 e il Novembre 2013.

\section{Risultati}

Abbiamo trovato sei pubblicazioni (7-12): tre studi randomizzati $(7,10,11)$ e tre review $(8,9,12)$. Uno degli studi ran- 
domizzati (7), condotto su 140 pazienti di uno stesso centro, dopo un anno di follow up mostrava una migliore sopravvivenza delle FAV (100\% vs $82 \%)$, un minor numero di interventi $(19 \%$ vs $39 \%)$ e una minore crescita degli aneurismi preesistenti ( $23 \%$ vs $67 \%$ ) per il gruppo BT; lo studio non evidenziava differenze nell'incidenza delle infezioni e nel tempo di sanguinamento post dialitico tra i due gruppi. Un altro studio randomizzato multicentrico, condotto su 70 pazienti, ha mostrato, nel gruppo BT, un maggior numero di infezioni e di ematomi e una maggiore percezione del dolore (10). Un terzo studio randomizzato canadese, condotto su 140 pazienti, non ha evidenziato differenze nella percezione del dolore e nel tempo di sanguinamento tra il gruppo BT e il gruppo di controllo; sono stati rilevati un maggior numero di ematomi nel gruppo di controllo e un maggior numero di infezioni locali e di sepsi da Staphylococcus Aureus nel gruppo BT (11).

Nella review di Grudzinski (8), che ha analizzato la letteratura su MEDLINE, Embase (Gennaio 1980-Giugno 2012) e CINAHL (Gennaio 1997-Giugno 2012), inerente a studi randomizzati e osservazionali sulla BT, sono stati identificati 23 articoli e 3 abstract; 3 erano dei trial open-label e gli altri studi osservazionali. Gli studi erano prevalentemente descrittivi ed erano basati sul confronto tra la BT e la tecnica a scala di corda. Secondo gli Autori, gli studi che analizzavano la sopravvivenza degli accessi, l'ospedalizzazione, la qualità della vita, il dolore e la formazione di aneurismi avevano degli importanti limiti metodologici. Un unico dato certo che emerge dalla review di Grudzinski è l'aumentato rischio di infezione per la BT. Un'altra review di un gruppo di nefrologi canadesi (9) ha analizzato su Embase e MEDLINE gli studi osservazionali e randomizzati dal 1990 al 2011 e gli atti di congressi e conferenze tra il 2007 e il 2011. Tra gli argomenti della review c'era il confronto tra la BT e la tecnica a scala di corda e tra l'uso e il non uso della pomata antibiotica nella BT. La BT era associata a un'elevata incidenza della batteriemia, che, in alcuni studi, era sovrapponibile a quella dei cateteri venosi centrali (CVC). L'impiego locale della mupirocina mostrava un'attenuazione delle infezioni. Nell'ultima review analizzata, del Novembre 2013 (12), gli Autori sottolineavano che la minore percezione del dolore rilevata dagli studi osservazionali non veniva confermata dagli studi randomizzati. Con la BT, veniva rilevata una minore incidenza di ematomi e aneurismi, bilanciata da un maggior numero di episodi infettivi locali e sistemici.

\section{Discussione}

Dall'analisi della recente letteratura, considerando i vari end point della BT, come dolore, formazione di aneurismi e sanguinamento post dialitico, non sembra emergere alcuna evidenza a favore della superiorità della BT. Per quanto riguarda la percezione del dolore, non vi sono dati certi. Come rilevato dalla review di Atkar (12), la minore percezione del dolore evidenziata dagli studi osservazionali non trovava conferma negli studi randomizzati. Per contro, nello studio randomizzato di Chow (10), la percezione del dolore era maggiore nel gruppo BT. Riguardo alla minore formazione di aneurismi con la BT, come evidenziato dallo studio di
Vaux (7), appare discutibile se a determinare gli aneurismi o il loro ingrandimento sia la modalità di venipuntura. È noto, infatti, che la formazione di un aneurisma è spesso sostenuta da una stenosi e che le stenosi non sono provocate solo dalla venipuntura. Diversi Autori concordano sul fatto che a determinare gran parte delle stenosi delle vene efferenti delle FAV siano meccanismi fisiopatologici legati all'emodinamica (13-15). Anche se le zone di venipuntura possono essere soggette a stenosi, la sede prevalentemente interessata dalle stenosi è quella juxta-anastomotica (16-18), generalmente poco utilizzata per la venipuntura.

Un dato certo e inequivocabile, dimostrato dalla maggior parte degli studi, osservazionali e randomizzati, è la maggiore incidenza di infezioni locali e sistemiche, legate alla difficoltà di disinfettare adeguatamente il tunnel sottocutaneo tra la cute e il vaso. Probabilmente, la lunga esperienza sui tunnel dei cateteri per dialisi peritoneale (CP) e dei CVC per emodialisi avrebbe potuto farci prevedere un aumento degli eventi infettivi. La creazione di un tunnel sottocutaneo, infatti, è fonte di un potenziale inquinamento per i germi che si possono annidare nel tunnel stesso. La cuffia di dacron dei CVC e dei CP, collocata all'estremità esterna del tunnel ha proprio il fine di esercitare una barriera protettiva contro l'ingresso dei germi, oltre che di ancorare il catetere al tessuto sottocutaneo. Nel tunnel creato dal buttonhole, la cuffia di dacron non c'è, per cui esso sembrerebbe maggiormente esposto alla colonizzazione batterica. Un fattore aggravante della $\mathrm{BT}$, rispetto alla staticità dei tunnel dei $\mathrm{CP}$ e dei $\mathrm{CVC}$, è rappresentato dallo scorrimento dell'ago che, nel suo tragitto, potrebbe portarsi dietro detriti cellulari e batteri eventualmente non rimossi dalla disinfezione e inocularli nel torrente circolatorio. È possibile che rigorosi protocolli di disinfezione con accurate manovre di prevenzione possano ridurre l'incidenza delle infezioni. Infine, nell'ottica che una FAV possa aver bisogno di "manutenzione" e di eventuali reinterventi, è verosimile ritenere la presenza del tunnel fibroso, che lega tenacemente la cute al vaso, un potenziale ostacolo all' isolamento chirurgico del vaso stesso ed al suo utilizzo per una nuova anastomosi.

Avendo evidenziato una serie di limitazioni della BT, come necessità di un training accurato, motivazioni dello staff $\mathrm{e}$ selezione dei pazienti, alcuni Autori consigliano la tecnica solo in casi complessi, quando la venipuntura di una FAV può risultare difficile (16). Personalmente, ritengo che l'utilizzo dell'ecoguida possa facilitare la puntura delle FAV complesse.

\section{Conclusioni}

Da questa analisi, non emergono dati sufficienti per indurci a intraprendere un'esperienza con la BT, e certamente non per scetticismo aprioristico, ma per scelta, probabilmente opinabile, razionale e motivata. Nella nostra pratica clinica quotidiana, abbiamo già sufficienti problemi con i CVC e non vi è alcuna necessità di incrementarne la prevalenza trasformando delle buone FAV in cateteri con la BT, che, in alcune esperienze, ha mostrato un'incidenza di fenomeni infettivi sovrapponibile a quella dei CVC (9). Crediamo che i rischi della BT possano essere giustificati solo in casi opportunamente selezionati (vasi particolarmente profondi o tratti troppo brevi), in 
cui la BT può facilitare l'utilizzo della FAV. Non si può escludere che ulteriori studi randomizzati e controllati possano, in futuro, produrre un giudizio diverso sulla BT.

\section{Riassunto}

La tecnica buttonhole (BT) è un'alternativa alle tecniche standard per l'incannulamento della fistola artero-venosa (AVF). Non è certo se i vantaggi della BT siano superiori ai rischi ad essa associati.

Al fine di analizzare la letteratura recente, abbiamo cercato su PubMed le voci "randomized controlled study on buttonhole" e "review of buttonhole cannulation" negli anni tra il Gennaio 2011 e il Novembre 2013.

Abbiamo identificato tre studi randomizzati e tre recensioni. Uno studio randomizzato ha mostrato una migliore sopravvivenza delle FAV (100\% vs $82 \%$ ), meno interventi ( $19 \%$ vs $39 \%$ ) e una minore crescita degli aneurismi preesistenti (23\% vs 67\%) nel gruppo BT. Un secondo studio ha mostrato un maggior numero di infezioni ed ematomi e una maggiore percezione del dolore nel gruppo BT. Nel terzo studio, non ci sono state differenze nella percezione del dolore e nel tempo di sanguinamento tra il gruppo BT e il gruppo di controllo e sono stati rilevati un maggior numero di ematomi nel gruppo di controllo e un maggior numero di infezioni locali e sepsi nel gruppo BT. Le tre review non hanno mostrato risultati certi riguardo agli end point della BT (percezione del dolore, formazione di aneurismi, tempo di sanguinamento), confermando l'aumentato rischio di infezioni locali e sistemiche.

Sulla base di questa analisi, si può concludere che la BT produce un aumento del rischio di infezioni locali e sistemiche senza benefici definiti. Studi recenti suggeriscono di riservare la BT a casi selezionati (vasi particolarmente profondi o tratti troppo brevi). Non si può escludere che ulteriori studi randomizzati e controllati possano produrre un diverso giudizio sulla BT.

Parole chiave: Accessi vascolari, Buttonhole, Incannulamento della fistola artero-venosa

Dichiarazione di conflitto di interessi: L'Autore dichiara di non avere conflitto di interesse.

Contributi economici agli Autori: L'Autore dichiara di non aver ricevuto sponsorizzazioni economiche per la preparazione dell'articolo.

\section{Indirizzo degli Autori:}

Dr. Marcello Napoli

U.O.C. Nefrologia e Dialisi

P.O. S. Caterina Novella

Via Roma

73013 Galatina (LE)

mar.napoli55@gmail.com

\section{Bibliografia}

1. Twardowski ZJ, Kubara H. Different sites versus constant sites of needle insertion into arteriovenous fistulas for treatment by repeated dialysis. Dial Transplant 1979; 8: 978-80.

2. Verhallen AM, Kooistra MP, van Jaarsveld BC. Cannulating in haemodialysis: rope-ladder or buttonhole technique? Nephrol Dial Transplant 2007; 22: 2601-4.

3. van Loon MM, Goovaerts T, Kessels AG, van der Sande FM, Tordoir JH. Buttonhole needling of haemodialysis arteriovenous fistulae results in less complications and interventions compared to the rope-ladder technique. Nephrol Dial Transplant 2010; 25 (1): 225-30.

4. Ball L, Treat L, Riffle V, et al. A multi-center perspective of the buttonhole technique in the Pacific Northwest. Nephrol Nurs J 2007; 34: 234-41.

5. Ball LK. Improving arteriovenous fistula cannulation skills. Nephrol Nurs J 2005; 32: 611-7.

6. Krönung G. Plastic deformation of Cimino fistula by repeated puncture. Nephrol Dial Transplant 1984; 13: 635-8.

7. Vaux E, King J, Lloyd S, et al. Effect of buttonhole cannulation with a polycarbonate PEG on in-center hemodialysis fistula outcomes: a randomized controlled trial. Am J Kidney Dis 2013; 62 (1): 81-8.

8. Grudzinski A, Mendelssohn D, Pierratos A, Nesrallah G. A systematic review of buttonhole cannulation practices and outcomes. Semin Dial 2013; 26 (4): 465-75.

9. Mustafa RA, Zimmerman D, Rioux JP, et al. Vascular access for intensive maintenance hemodialysis: a systematic review for a Canadian Society of Nephrology clinical practice guideline. Am J Kidney Dis 2013; 62 (1): 112-31.
10. Chow J, Rayment G, San Miguel S, Gilbert M. A randomised controlled trial of buttonhole cannulation for the prevention of fistula access complications. J Ren Care 2011; 37 (2): 85-93.

11. MacRae JM, Ahmed SB, Atkar R, Hemmelgarn BR. A randomized trial comparing buttonhole with rope ladder needling in conventional hemodialysis patients. Clin J Am Soc Nephrol 2012; 7 (10): 1632-8.

12. Atkar RK, Macrae JM. The buttonhole technique for fistula cannulation: pros and cons. Curr Opin Nephrol Hypertens 2013; 22 (6): 629-36.

13. Roy-Chaudhury P, Kelly BS, Zhang J, et al. Hemodialysis vascular access dysfunction: from pathophysiology to novel therapies. Blood Purif 2003; 21: 99-110.

14. Roy-Chaudhury P, Samaha A, Zhang J, et al. Aggressive early neointimal hyperplasia contributes to AV fistula maturation failure. $\mathrm{J}$ Am Soc Nephrol 2006; 17: F-PO1056.

15. Meyerson SL, Skelly CL, Curi MA, et al. The effects of extremely low shear stress on cellular proliferation and neointimal thickening in the failing bypass graft. J Vasc Surg 2001; 34: 90-7.

16. Rajan DK, Bunston S, Misra S, Pinto R, Lok CE. Dysfunctional autogenous hemodialysis fistulas: outcomes after angioplasty are there clinical predictors of patency? Radiology 2004; 232 (2): 508-15.

17. Clark TW, Hirsch DA, Jindal KJ, Veugelers PJ, LeBlanc J. Outcome and prognostic factors of restenosis after percutaneous treatment of native hemodialysis fistulas. J Vasc Interv Radiol 2002; 13 (1): 51-9.

18. Tessitore N, Mansueto G, Lipari G, et al. Endovascular versus surgical preemptive repair of forearm arteriovenous fistula juxtaanastomotic stenosis: analysis of data collected prospectively from 1999 to 2004. Clin J Am Soc Nephrol 2006; 1: 448-54. 